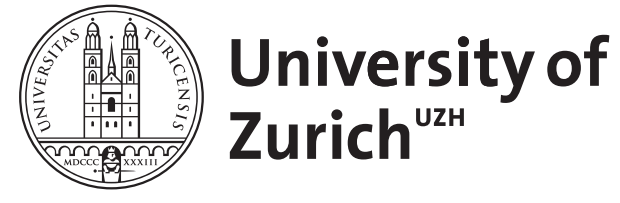

\title{
Philosophie der Kunst
}

\author{
Räber, Michael Ivo
}

\begin{abstract}
Für die dominanten ästhetischen Theorien seit Kant ist das Ästhetische charakterisiert durch Interesse- und Zwecklosigkeit. Für den Pragmatismus sind das Praktische und das Funktionale hingegen ernst zu nehmende philosophische Konzepte. Angesichts dieser Ausgangslage wäre eine ernsthafte Beschäftigung mit dem Ästhetischen im Pragmatismus eigentlich nicht zu erwarten. Indessen hat der Pragmatismus die Relevanz des Ästhetischen für die Philosophie seit langem anerkannt. Genauso wie der Pragmatismus eine vielstimmige Denktradition ist, gilt dies auch für die pragmatistische Ästhetik. Dennoch lassen sich bei unterschiedlichen pragmatistischen Denkern einige Schlüsselbegriffe zur Ästhetik identifizieren. Der vielleicht wichtigste ist der Begriff 〉Erfahrung〈, der sich inhaltlich weit über das Gebiet der Künste hinaus verzweigt. Neben dem Begriff der Erfahrung sind es die Themen Naturalismus, Kontinuität des Ästhetischen mit dem Praktischen, kultureller Perfektionismus und soziale Transformation sowie Demokratisierung der Kunst, welche im klassischen Pragmatismus und im Neopragmatismus bei der Reflexion über Kunst und Ästhetik eine zentrale Rolle einnehmen. Während John Dewey im 1934 erschienen Buch Art as Experience als erster der klassischen Pragmatisten eine ausgereifte Kunstphilosophie formuliert hat, lassen sich bereits vor Dewey Spuren einer pragmatistischen Beschäftigung mit dem Ästhetischen auffinden, und zwar bei Ralph Waldo Emerson, Charles Sanders Peirce und William James
\end{abstract}

DOI: https://doi.org/10.1007/978-3-476-04557-7_23

Posted at the Zurich Open Repository and Archive, University of Zurich

ZORA URL: https://doi.org/10.5167/uzh-153354

Book Section

Published Version

Originally published at:

Räber, Michael Ivo (2018). Philosophie der Kunst. In: Festl, Michael G. Handbuch Pragmatismus. Stuttgart: J.B. Metzler Verlag, 171-177.

DOI: https://doi.org/10.1007/978-3-476-04557-7_23 


\section{Philosophie der Kunst}

\section{Ästhetik bei Emerson, Peirce und James}

Für die dominanten ästhetischen Theorien seit Kant ist das Ästhetische charakterisiert durch Interesseund Zwecklosigkeit. Für den Pragmatismus sind das Praktische und das Funktionale hingegen ernst zu nehmende philosophische Konzepte. Angesichts dieser Ausgangslage wäre eine ernsthafte Beschäftigung mit dem Ästhetischen im Pragmatismus eigentlich nicht zu erwarten. Indessen hat der Pragmatismus die Relevanz des Ästhetischen für die Philosophie seit langem anerkannt.

Genauso wie der Pragmatismus eine vielstimmige Denktradition ist, gilt dies auch für die pragmatistische Ästhetik. Dennoch lassen sich bei unterschiedlichen pragmatistischen Denkern einige Schlüsselbegriffe zur Ästhetik identifizieren. Der vielleicht wichtigste ist der Begriff `Erfahrung «, der sich inhaltlich weit über das Gebiet der Künste hinaus verzweigt. Neben dem Begriff der Erfahrung sind es die Themen Naturalismus, Kontinuität des Ästhetischen mit dem Praktischen, kultureller Perfektionismus und soziale Transformation sowie Demokratisierung der Kunst, welche im klassischen Pragmatismus und im Neopragmatismus bei der Reflexion über Kunst und Ästhetik eine zentrale Rolle einnehmen.

Während John Dewey im 1934 erschienen Buch Art as Experience als erster der klassischen Pragmatisten eine ausgereifte Kunstphilosophie formuliert hat, lassen sich bereits vor Dewey Spuren einer pragmatistischen Beschäftigung mit dem Ästhetischen auffinden, und zwar bei Ralph Waldo Emerson, Charles Sanders Peirce und William James.

Trotz der Bezeichnung >Transzendentalphilosoph gilt Emerson mittlerweile auch als wichtiger Vorläufer des klassischen Pragmatismus (s. Kap. 5). Er hat einige der Themen vorweggenommen, die vor allem Dewey in seiner Ästhetik eingehend beschäftigt haben. Im Essay »Art« von 1841 werden zwei normative Standpunkte thematisiert, die sich später bei Dewey wiederfinden. Zum einen hat Emerson der Kunst eine zentrale Rolle bei der individuellen Selbsterschaffung durch Erziehung zugewiesen. Kunst sollte für Emerson immer auch erzieherisch sein, insofern sie unausgereift bleibt, »if it is not practical and moral, if it do[es] not stand in connection with the conscience, if it do[es] not make the poor and uncultivated feel that it addresses them with a voice of lofty cheer « (1987, 215). Zum anderen, und damit zusammenhängend, hat Emerson Kunst nicht vom Praktischen abgetrennt, sondern beides als wesentlich miteinander verbunden gesehen. Ist diese Verbundenheit nicht gegeben, verkommen Kunst und ästhetische Erfahrungen zu einer Flucht vom Leben, zu einem Eskapismus: »As soon as beauty is sought not from religion and love, but for pleasure, it degrades the seeker« (ebd.). Dies zu verhindern ist nur möglich, wenn das Leben selbst wie ein Kunstwerk von uns selbst erschaffen wird, und am besten wird uns das gelingen, wenn wir Kunst als alltägliche Praxis verstehen. Alltagserfahrungen wären dann nicht kategorial getrennt von ästhetischen Erfahrungen, und es ginge darum, sie möglichst mit ästhetischer Qualität aufzuladen.

Peirce, der Vater des Pragmatismus und der Semiotik, hat wichtige Beiträge zur Theorie der Symbole und Interpretation beigesteuert, welche immer noch Bedeutung haben in der philosophischen Ästhetik. Seine Semiotik als Theorie der Zeichen kann als eine bruchstückhafte Kulturphilosophie verstanden werden (s. Kap. 13). Er hat darüber hinaus in seiner Ontologie auch die unmittelbar gefühlte Qualität von Erfahrung hervorgehoben - welche zentral ist in der Ästhetik mittels seiner ersten Kategorie der `Erstheit $<$ (firstness) (s. Kap. 1). Peirce hat auch auf der Kontinuität zwischen Ästhetik und Ethik insistiert; er ging sogar so weit, "Ethics dependent upon Esthetics zu machen $(1998,142)$ und hat das moralische Gute "as a particular species of the esthetically good " (ebd., 201) bezeichnet. Während die Ästhetik neben der Ontologie, der Logik, der Ethik und der Metaphysik für Peirce' Werk relevant war, gibt es bei ihm aber keine bedeutende systematische Abhandlung über Ästhetik.

Obgleich William James kein Interesse daran hatte, über Kunstphilosophie philosophisch nachzudenken oder zu schreiben, maß er der ästhetischen Dimension von Erfahrung (der Erfahrungsqualität) große Bedeutung zu. In seinem Buch von 1890 The Principles of Psychology, also in einem Werk, das sich nicht mit Kunst und Ästhetik im engeren Sinne auseinandersetzt, skizziert er bereits einige wichtige Themen der pragmatistischen Ästhetik, die dann von Dewey gut 40 Jahre später in Art as Experience noch viel detaillierter ausgearbeitet worden sind. Drei solche Themen sind zu nennen (vgl. Shusterman 2011). Erstens die Naturalisierung des Ästhetischen: Kunst und ästhetische Erfahrungen sind materialisierte Äußerungen von natürlichen Kräften, die aus der Interaktion von Menschen mit ihren natürlichen und kulturellen Umwelten entstehen und in ihnen gefiltert werden. Zweitens ein ästhetischer Meliorismus bzw. Perfektionis- 
mus. Der ultimative Zweck alles Ästhetischen sollte die Verbesserung der Erfahrungsqualität sein: »The world of aesthetics and ethics is an ideal world, a Utopia, a world which the outer relations persist in contradicting, but which we as stubbornly persist in striving to make actual« (James 1983, 1235). Drittens der bereits bei Emerson thematische Zusammenhang des Ästhetischen und des Praktischen: Ästhetische Erfahrungen und Kunst sollten in allen Lebensbereichen ihren Platz haben und nicht nur in klar abgetrennten Institutionen.

\section{John Deweys Ästhetik}

Dewey hat seine ästhetische Theorie erst gegen Ende seines Lebens im Buch Art as Experience von 1934 festgehalten, skizzierte aber einige seiner Ideen zur Ästhetik bereits im früheren Werk Experience and $\mathrm{Na}$ ture von 1925. Seine metaphysische Analyse der Interaktion von Erfahrung und Natur, die er im früheren Werk ausbreitet, weist in mancher Hinsicht voraus auf seine späteren Einsichten bezüglich des Begriffs der ästhetischen Erfahrung in seiner ästhetischen Theorie. In Experience and Nature (2007) entwickelt Dewey einen Kulturbegriff (Erfahrung und Kultur sind darin begrifflich synonym), für den sowohl Erfahrung wie auch von Menschen erschaffene Sinn- und Bedeutungszuschreibungen als Kulturprodukte zu verstehen sind - was zweifellos auch Kunstprodukte und Kunsterfahrungen mit einschließt (s. Kap. 13). Die eigentliche Ästhetik von Dewey, wie er sie dann in Art as Experience entwickelt hat, lässt sich aufschlüsseln anhand der eingangs genannten zentralen Begriffe: Ästhetische Erfahrung, Naturalismus, Kontinuität des Ästhetischen mit dem Praktischen, kultureller Perfektionismus und soziale Transformation sowie Demokratisierung der Kunst. Das vermutlich wichtigste Thema in Deweys Ästhetik ist die ästhetische Erfahrung. Das eigentliche Kunstwerk ist für Dewey nicht das von einem Künstler hergestellte oder von einem Rezipienten betrachtete materielle Kunstobjekt, sondern es besteht in den Erfahrungen, die der Künstler bzw. der Rezipient mit diesem materiellen Objekt macht. Kunst zeigt sich nicht nur in ästhetischen Erfahrungen, sondern ist eigentliche ästhetische Erfahrung, behauptet Dewey. Was aber ist ästhetische Erfahrung? Für Dewey ist ästhetische Erfahrung eine intensivierte, gutgeordnete, unmittelbar erfüllende Erfahrung, ein Gefühl, das heraussticht aus dem alltäglichen Erfahrungsstrom als etwas Spezielles - als eine Erfahrung: intensiv, einheitlich, markant und einprägsam. Ästhetische Erfahrung kann auf eine Weise überwältigend, unmittelbar situiert und also gegenwärtig sein. Mit Unmittelbarkeit kann »eine dunkle Landschaft von einem Blitz erhellt « (Dewey 1980, 33) gemeint sein, zu der nicht nur das momentane Erkennen eines vertrauten Gegenstandes gehört, sondern ebenso die anhaltende Präsenz eines zeitlich gedehnten visuellen Prozesses, der auch das Resultat früherer Erfahrungen ist. Die vermeintliche Unvermitteltheit jeder besonderen ästhetischen Erfahrung ist deshalb in Isolation bedeutungslos, aber bedeutungsgeladen vor dem Hintergrund eines diachronen Erfahrungsstroms. Ästhetische Erfahrung ist also unmittelbar und subjektiv, dabei aber immer schon verwiesen auf Sinn- und Bedeutungshorizonte sozialer Zeichenund Interaktionsprozesse einer bestimmten Kultur.

Obwohl Kunst folglich ein Kulturprodukt ist, besteht Dewey darauf, Kunst ebenso sehr als in der natürlichen Welt verwurzelt zu sehen, in den grundlegenden Bedürfnissen, Wünschen und Rhythmen des mit dieser Welt interagierenden menschlichen Organismus. Für Dewey unterliegt den Rhythmen jeder Art von Kunst und jeder Art von Kunstwerken das Grundmuster der Interaktion des lebendigen Geschöpfs mit seiner gegebenen Umwelt. Jeder noch so profanen Art von Erfahrung liegt im Kern ein ästhetischer Impuls im Rohzustand zugrunde; Erfahrung selbst ist »die Keimzelle der Kunst« (ebd., 28). Wir können uns dies vergegenwärtigen, so Dewey, wenn wir beispielsweise ein Holzfeuer im Kamin anfachen und dabei den emporsteigenden Flammen zusehen, während wir in der Glut stochern. Der praktische Grund des Stocherns liegt auf der Hand, wir wollen, dass das Feuer besser brennt. Das dabei gezeigte Engagement und die erlebte Faszination aber konstituieren unsere alltäglichen und unausgereiften ästhetischen Erfahrungen. Dieses Beispiel zeigt: Kunstprodukte sind nur in einer abstrahierten Form externe Objekte, die wir distanziert aus einer Beobachterperspektive kühl registrieren, zumeist aber bestehen sie in unseren Erfahrungen selbst, die wir als schöpferische Beteiligte unweigerlich machen.

Ästhetische Erfahrungen sind also nicht kategorial getrennt von Alltagserfahrungen, sondern stehen in einer Kontinuität mit ihnen, insofern sie gesteigerte und ausgereifte Formen dieser in allen Erfahrungen angelegten Keime sind. Diese Naturalisierung ästhetischer Erfahrung impliziert für Dewey auch die $\mathrm{Zu}$ rückweisung der herkömmlichen dichotomischen Trennung von ästhetisch/praktisch, wie sie in der kan- 
tischen Ablehnung der Funktionalität des Ästhetischen zugunsten der Wertschätzung einer reinen ästhetischen Form angelegt ist. Mit Funktionalität des Ästhetischen ist bei Dewey zweierlei gemeint. Erstens hat Kunst für Dewey die Funktion, ästhetische Erfahrungen $\mathrm{zu}(\mathrm{re})$ konstruieren, also Objekte zu kreieren, welche unsere Fähigkeit, bedeutungsvolle und wertschätzende Erfahrungen $\mathrm{zu}$ machen, verstärken. Zweitens hat sie die Funktion, uns dabei zu helfen, sinnstiftende Kategorien dafür zu entwickeln, wie wir Dinge ganz allgemein erfahren.

Pragmatisten versuchen nicht nur einfach die Realität zu verstehen, sie wollen sie darüber hinaus (und vielleicht in erster Linie) verbessern. Dieser perfektionistische Grundzug des Pragmatismus spiegelt sich auch in der ästhetischen Theorie von Dewey wider und zwar in der Weigerung, sich aufbloß formale Definitionen oder abstrakte Wahrheiten über Kunst und Schönheit zu beschränken. Vielmehr ist seine Kunstphilosophie bemüht, uns begriffliche Instrumente in die Hand zu geben, wie wir die Erfahrungsqualität von Kunst und anderen ästhetischen Phänomenen verbessern können. Perfektionismus bezieht sich bei Dewey auch auf die Idee, dass wir vorhandene individuelle praktische Gewohnheiten (`habits`) oder kollektive Bräuche (`customs $\triangleleft$ ) nicht einfach so akzeptieren sollten, sondern dass wir als Individuum und als Gemeinschaft bestrebt sein sollten, bessere Gewohnheiten auszubilden, als die bestehenden es sind. Kunst zu machen und zu reflektieren stehen dabei für Dewey modellhaft für solche Bestrebungen. Imagination, schreibt er, »ist das wichtigste Instrument des Guten « und »Kunst moralischer als die Sittenlehren« (ebd., 401). Imagination ist in beispielhafter Weise konstituierend für Kunst. Daraus folgt auch, dass Kunst ein exemplarisches Mittel für die Perfektionierung menschlichen Lebens ist. Das hat u. a. damit zu tun, worauf Thomas Alexander hingewiesen hat, dass für Dewey das Herstellen von Bedeutung mit den Mitteln der Kunst »the ongoing process of trying to make sense « (1987, xviii) ist - es ist der Versuch, dem gegenwärtigen Material der praktischen und sozialen Lebensumstände Sinn abzugewinnen und neuen Sinn zu verleihen.

Solch kultureller Perfektionismus und das Streben nach sozialer Transformation gehen bei Dewey aber weder auf im konservativen Wunsch nach einer Rückkehr in die Vergangenheit noch in einer Affirmation der künstlerischen Moderne. Geht es dem Ersteren um die nostalgische und letztlich unbegründete Wiederherstellung vergangener sinnstiftender Praktiken, besteht die Pointe für Teile der künstlerischen Moder- ne im Imperativ, Sinn gegen Form einzutauschen. Deweys Vorstellung der Perfektionierung des menschlichen Lebens durch Ästhetik hingegen ist weder rückwärtsgewandt noch verzichtet sie auf die Hoffnung, Sinn herstellen zu können.

Bestandteil der Idee kultureller und sozialer Perfektionierung ist die Idee der Demokratisierung der Kunst. Damit ist mit Dewey die Ausweitung des Kunstbegriffs über den zu eng gefassten Begriff der schönen Künste hinaus gemeint, welche die Erfahrungen und Tätigkeiten von noch mehr Menschen, von noch mehr Klassen und sozialen Schichten umfassen soll. Die Zielscheibe von Dewey ist hier offensichtlich: jene Ansicht über Kunst, welche »die Kunst auf einen entrückten Sockel « $(1980,12)$ ins Museum verbannt:

\begin{abstract}
"Philosophische Theorie hat sich nur auf solche Arten von Kunst eingelassen, die Stempel und Siegel der Anerkennung durch eine soziale Schicht trugen, die Rang und Autorität hatte. Volkskünste konnten blühen, doch man schenkte innen keine literarische Aufmerksamkeit« (ebd., 217).
\end{abstract}

Die Ausweitung des Kunstbegriffs hat also eine konkret politische Bedeutung. Dewey hat seine ästhetische Theorie auf ein umfassendes Ideal des demokratischen Lebens hin weitergedacht, auf eine Philosophie der Zivilisation und Kultur. Die wahrhaftig demokratische Gesellschaft strebt nach der Befreiung von Erfahrung, d. h., nach einer Vermehrung von intensivierten ästhetischen Erfahrungen. Damit dies möglich wird, müssen institutionelle Rahmenbedingungen geschaffen werden, welche das zulassen und fördern: soziale, erzieherische und politische Institutionen. Dies kann für Dewey aber nur geschehen, wenn ästhetische Erfahrungen nicht nur im engen Bereich der schönen Künste möglich sind, sondern in allen lebensweltlichen Bereichen. Solange also die Trennung zwischen den schönen Künsten und lebensweltlichen ästhetischen Erfahrungen bzw. die Trennung zwischen dem Schönen und dem Nützlichen weiter besteht, kann das keine Wirklichkeit werden. Die Geschichte der strengen Trennung des Nützlichen vom Schönen, so Dewey, ist auch die Geschichte der Industrialisierung, im Zuge deren sich die Arbeits- und Produktionsprozesse zu einer Art »aufgeschobene[s] Leben « und der Konsum zu einem "verordneten Genuss der Früchte fremder Arbeit« entwickelt haben (ebd., 37). In einer mangelhaften Zivilisation wird Kunst dazu gemacht und dazu gebraucht, den hauptsächlichen Tätigkeiten des Lebens zu entfliehen und 
ist bloß»deren nebensächliche Verzierung ( (ebd., 97). Freilich war die Trennung zwischen den schönen und den nützlichen Künsten schon den Griechen bekannt, aber durch die industrielle Revolution wurde sie verstärkt - durch Massenproduktion und Mechanisierung der Arbeitswelt. Die Trennung von schöner Kunst und nützlichem Handwerk spiegelt sich für Dewey also in den industriellen und ökonomischen Verhältnissen seiner Zeit, beispielsweise auch in der Trennung von Arbeit und Freizeit, welche vorsieht, dass die ästhetische Erfahrungsqualität für den Bereich der Freizeit vorgesehen ist, nicht aber für den Bereich der Arbeit. Nur ein radikaler sozialer Wandel, der mehr Beteiligung der Arbeiter an der Produktion und bei der Verteilung von Gütern mit sich führe, war Dewey überzeugt, würde dies ändern können. Ein verstärktes Gefühl für Freiheit und eine größere Partizipation in den Produktionsprozessen würde den Arbeitern ein unmittelbares Interesse und ästhetische Befriedigung durch die Arbeit verschaffen. Wenn aber Kunst nur der »Schönheitssalon einer Zivilisation« (ebd., 396) bleibt, verharren sowohl Kunst wie auch Zivilisation in einem unvollkommenen Zustand.

\section{Ästhetik im Neopragmatismus}

Der pragmatistische Imperativ, das Alltägliche mit dem Ästhetischen zusammenzudenken, hat sich in der philosophischen Reflexion über Ästhetik seit Dewey auf unterschiedliche Weise niedergeschlagen. Auch wenn sich Autoren wie der späte Wittgenstein oder J.L. Austin nicht als Erben des klassischen Pragmatismus verstehen würden, sind sie ihm inhaltlich nahe, jedenfalls was die Hinwendung zur Alltagsphilosophie angeht. In Stanley Cavells ästhetischen Schriften (Cavell 1988; 1990) findet dieser Imperativ eine besonders relevante Stimme. Auch bei Arthur C. Danto, einem eher analytisch ausgerichteten Kunsttheoretiker, findet sich eine Beschäftigung mit der Ästhetik des Alltäglichen, im Zusammenhang mit der Frage nach dem Verhältnis von Kunstobjekten und alltäglichen Objekten - auch wenn Danto vorrangig am Unterschied zwischen Kunstobjekten und alltäglichen Objekten interessiert ist (vgl. Danto 1981; 2014).

Die im engeren Sinne pragmatistische Ästhetik ist in der Mitte des 20. Jahrhunderts von der Agenda der philosophischen Ästhetik mehr oder weniger verschwunden. Analytische Theoretiker der Ästhetik haben Deweys Ästhetik als ein Sammelsurium vager, inkonsistenter und ungeordneter Spekulationen ver- worfen - hauptsächlich wohl deshalb, weil die ästhetische Theorie der klassischen Pragmatisten bewusst keine hinreichenden und notwendigen Bedingungen für die Definition von Kunstobjekten formuliert hat. Außerhalb der in einem engeren Sinne verstandenen analytischen Kunsttheorie hat Deweys Ästhetik aber in neuerer Zeit detaillierte Analyse und kreative Erläuterungen erhalten durch Philosophen wie John J. McDermott, Thomas Alexander, und Crispin Sartwell. Erwähnenswert ist hier auch Gregory Pappas' (2008) Studie zu den engen Verflechtungen von Deweys Ästhetik mit seiner Ethik und seinen demokratietheoretischen Überlegungen.

Andererseits gibt es eine Handvoll Philosophen, die der analytischen Philosophie mehr oder weniger nahe stehen und zentrale Ideen der Ästhetik der klassischen Pragmatisten dazu benutzt haben, neue Ansätze sowohl zu herkömmlichen Themen der Ästhetik wie auch zu zeitgenössischen Themen zu entwickeln, beispielsweise zur Ästhetik der Massenmedien oder zur Ästhetik der Kunst des Lebens. Autoren wie Joseph Margolis, Nelson Goodman, Richard Rorty und Richard Shusterman haben pragmatistische Ideen aufgegriffen, um zu zeigen, wie die Interpretation von Kunstwerken sinnvoll sein kann, ohne die Notwendigkeit von starren Entitäten anzunehmen, welche diesen Interpretationen als gleichbleibende Objekte zugrunde liegen. Sie versuchen folglich zu erklären, wie es möglich ist, dass wir gemeinsam sinnvoll über Kunstwerke sprechen können - vermittelt durch einen gemeinsamen diskursiven Bezugsrahmen des Sprechens über Kunstwerke im Kontext von traditionell verankerten aber diskursiv offenen Praktiken -, ohne dabei eine fixe, substantielle Natur von Kunstwerken, welche diesen erst eine beständige Identität verleihen und eine gültige Interpretation ermöglichen würde, vorauszusetzen.

Dieses Bemühen ist bei den genannten Denkern unterschiedlich ausgeprägt; sie alle teilen aber die Auffassung, dass der Sinn und die Bedeutung von Kunstwerken nicht getrennt von deren historischen und kulturellen Entstehungs- und Rezeptionskontexten erfasst werden können. Margolis hat diese Überlegung als Ausgansunkt einer feingliedrigen Metaphysik kultureller Objekte genommen (1984; 2010; 2016). In einer Reihe von kürzlich erschienenen Publikationen hat er explizit den Dialog mit den klassischen Pragmatisten und deren neopragmatistischen Nachfahren aufgenommen, im Speziellen mit Richard Rorty und Hilary Putnam. In On Aesthetics (2008) behandelt er Kunst in einer Weise, die Ähnlichkeiten zu Dewey aufweist. Für 
Margolis ist ein Kunstwerk eine »historicized utterance that is physically embodied, culturally emergent, possessing Intentionally qualified properties that are determinable but not determinate in the way mere material properties are said to be« (ebd., 136).

Indem Margolis Kunstwerke so definiert, versucht er einen Mittelweg zu finden zwischen idealistischen und reduktionistisch-materialistischen Kunsttheorien. Diesem anti-dualistischen Ansatz zufolge durchdringt Kultur die materielle Welt und lädt sie mit Bedeutung auf. Eben darin liegt die Unmöglichkeit einer statisch-fixierten Natur des Kunstwerks und auch der sie hervorbringenden Künstler. Bedeutung entsteht in den interpretativen Praktiken der historisch situierten Hersteller und Rezipienten von Kunst. Ästhetische Eigenschaften sind dabei nie einfach so gegeben oder aufgefunden, sondern kulturell konstruiert.

Goodman, ein weiterer Denker aus der zweiten Hälfe des 20. Jahrhunderts mit Affinitäten zum Pragmatismus, hat die Kontinuitätsthese Deweys aufgenommen und auf das Verhältnis von Kunst und Wissenschaft angewendet. Goodman lehnt ganz im Sinne des Pragmatismus eine Ästhetik ab, die den Wert von ästhetischen Objekten unabhängig von ihrer Funktionalität im reinen Genuss ihrer Form sehen will und betont dagegen die grundlegende Einheit von Kunst und Wissenschaft durch ihre gemeinsame kognitive Struktur. Im Weiteren ist er genau wie Dewey der Auffassung, dass letztlich nicht das gegenständliche Kunstwerk im Zentrum der ästhetischen Reflexion stehen sollte, sondern dessen Funktionalität in der dynamischen Erfahrung. Die Frage danach, was Kunst ist, sollte deshalb für Goodman durch die Frage ersetzt werden, wann Kunst ist $(1968 ; 1978 ; 1984)$. Goodman steht den klassischen Pragmatisten auch in einem anderen Zusammenhang nahe, nämlich in seiner Kritik an einem zeitgenössischen Museumsverständnis, welches Museen als Institutionen auffasst, die hauptsächlich dem Freizeitvergnügen dienen, anstatt sie als zentrale Bildungsinstitutionen zu verstehen, welche helfen könnten, die Art und Weise zu transformieren, wie wir die Welt sehen und interpretieren. Letzteres wird aber nur gelingen, so Goodman, wenn Museen Kunstwerke auf eine Weise wirksam werden lassen, dass sie "with all our experience and all our cognitive processes « interagieren, indem » $[\mathrm{r}]$ everberations from a work [...] travel in cycles through our everyday environment, other works, and itself, again and again, with ever-changing effect « $(1984,180)$.

Rorty hat eine ästhetische Interpretation von Themen aus dem klassischen Pragmatismus vorgelegt.
Zweifellos hat sich Rorty kaum zu ästhetischen Themen wie der Frage geäußert, was ein Kunstwerk ist oder worin ästhetische Erfahrung besteht (hat er doch den Erfahrungsbegriff generell abgelehnt), noch hat er eine Kunsttheorie mit einem Allgemeingültigkeitsanspruch entwickelt, was natürlich in seinen eigenen philosophie-methodischen Überzeugungen wurzelt. Dennoch hat sich Rorty in Kontingenz, Ironie und Solidarität (1989) positiv auf Deweys Ästhetik bezogen, um für das ästhetische Leben als eine Ethik der SelbstVervollkommnung und Selbst-Erschaffung zu argumentieren. Scott Stroud (2011) hat diese von Dewey inspirierte Idee der ästhetisch-ethischen Selbst-Kultivierung von Rorty weiterentwickelt.

Shusterman hat in seinem Buch Pragmatist Aesthetics (2000) einen detailreichen kritischen Kommentar zu Deweys Kunsttheorie verfasst und darauf aufbauend eine eigene Version der pragmatistischen Ästhetik entwickelt. Shusterman teilt viele von Deweys Grundintuitionen, die Dewey in Art as Experience angetrieben haben. Ebenso wie Dewey will er der Ästhetik eine prominentere Rolle in der Philosophie zuweisen gegenüber der als zu eng empfundenen Rolle, welche sie in der modernen Philosophie in vielen Teilen eingenommen hat. Dieses innerphilosophische Ziel ist eng verknüpft mit gesellschaftlichen Idealen: der Demokratisierung von Kunst und ihrer Wertschätzung im Alltag; dem melioristischen Potential von Kunst und von Kunsterfahrungen; sowie der radikalen Kritik an der Museumskunst. Neben diesen geteilten Zielen und Idealen geht Shusterman aber weiter als Dewey, wenn er argumentiert, dass die anhaltende Relevanz pragmatistischer Ästhetik von ihrer Bereitschaft und Fähigkeit abhängt, zeitgenössische ästhetische Phänomene in ihrer Theorie zu reflektieren, Phänomene, die zumeist aus der sich schnell wandelnden Populärkultur hervorgehen, welche ja gemäß Dewey gerade auch Gegenstand der ästhetischen Reflexion sein sollten (Shusterman 2006). Shusterman befürchtet, dass ohne eine nachhaltige Auseinandersetzung mit der Populärkultur die Kritik an der Museumskunst letztlich leer bleiben muss und auch das Ziel verfehlt wird, die stereotype Dichotomie hohe Kunst/ populäre Kunst zu lockern. Er hat sich deshalb aktiv mit einer Vielzahl von populären Künsten und ästhetischen Tätigkeiten auseinandergesetzt: Neben dem Einbezug von Rap Musik in Pragmatist Aesthetics, hat er sich u. a. mit Country-, Rock- und Technomusik, mit urbaner Ästhetik und mit körperlichen ästhetischen Phänomenen auseinandergesetzt. Für Letztere hat er den Begriff der somaesthetics geprägt, den er 
versteht als »the critical study and meliorative cultivation of how we experience and use the living body (or soma) as a sight of sensory appreciation (aesthesis) and creative self-fashioning « $(2008,1)$.

Auch wenn Shusterman Deweys Konzept der ästhetischen Erfahrung positiv rezipiert und weiterentwickelt hat, sieht er dennoch Grenzen. In erster Linie bemängelt er dessen Erklärungskraft, weil Dewey ästhetische Erfahrung letztlich als undefinierbar (und damit sprachlich nicht fassbar) versteht, trotz seinen Bemühungen, dem Konzept klar sichtbare Konturen zu verleihen. Wenn ästhetische Erfahrung aber wesentlich stumm und nicht in Sprache übersetzbar ist, verliert der Begriff seine Relevanz für das kritische Erläutern, Evaluieren und Urteilen über Kunst - Ästhetik als kritisch-normatives Unterfangen wird dann unmöglich. Hier kann Shusterman nur zugestimmt werden: Sofern (Kunst-)Kritik einen diskursiven Raum für jene egalitäre Kunstkonzeption bieten soll, wie sie Dewey vorschwebte, sind verständigungsorientierte Diskurse notwendig, in denen neue Kunstphänomene in breiten Teilen der Gesellschaft reflektiert werden können.

\section{Ausblick}

Pragmatistische Zugänge zur Ästhetik entwickelten sich seit den 1980er Jahren auch außerhalb des Neopragmatismus. Eine Fülle von neuen Forschungsfeldern innerhalb der philosophischen Ästhetik haben sich entwickelt, u. a. im Bereich der Ästhetik der Umwelt, der feministischen Ästhetik, der Ästhetik von alltäglichen Objekten, und somaesthetics, die sich inhaltlich mit vielen Themen der Ästhetik des klassischen Pragmatismus verknüpfen lassen. Die Diskussionen über Themen der hier skizzierten pragmatistischen Ästhetik sind also noch nicht zu einem Ende gelangt - innerhalb der pragmatistischen Literatur nicht, aber auch in gegenwärtigen außer-pragmatistischen Ästhetikdiskursen nicht.

Zwei der genannten Inhalte stehen momentan besonders im Fokus der Aufmerksamkeit: Einerseits die Ausdehnung des Ästhetikbegriffs über die Philosophie der schönen Künste hinaus auf die Beschäftigung mit Alltagsphänomenen, andererseits die enge Verbindung der Ästhetik mit dem Praktischen. Insbesondere die Debatte um die Ästhetik alltäglicher Phänomene hat in den letzten Jahren Fahrt aufgenommen. Die meisten Autoren, die sich an dieser Debatte beteiligen, sind von Deweys Ästhetik inspiriert, weichen aber von ihr auch ab. Innerhalb der Alltagsästhetik hat beispielsweise Arnold Berleant eine Unterkategorie etabliert, die er Sozialästhetik nennt und bei der es darum geht, zu erkennen, dass die Ästhetik eine entscheidende Rolle dabei spielt, den moralischen Charakter von Handlungen, Personen und menschlichen Interaktionen festzulegen (z. B. Berleant 2016).

Sowohl für diese Unterkategorie wie auch für die Alltagsästhetik als Ganzes gilt, dass sie die ästhetische Dimension menschlichen Handelns in den Blick nimmt. Zu nennen wäre hier beispielsweise die Ästhetik des Essens, bei der es aus diesem Blickwinkel nicht nur um die ästhetische Qualität des Produkts geht, sondern vielmehr um die Erfahrung des Essens oder um die Aktivität des Kochens selbst. Zweifellos ist diese Art von ästhetischer Theorie mit derselben Schwierigkeit konfrontiert wie Deweys Konzeption der ästhetischen Erfahrung auch - es wird schwierig, darüber einen kritischen und sinnvollen Diskurs zu führen. Es wird die Aufgabe künftiger pragmatistischer Reflexion sein, aufzuzeigen, wie wir beispielsweise ein sinnvolles Gespräch darüber führen können, ob die subjektive Erfahrung des Kochs oder des Speisenden tatsächlich eine wahrhaftig ästhetische Qualität aufweist oder nicht.

\section{Literatur}

Alexander, Thomas M.: John Dewey's Theory of Art, Experience, and Nature. The Horizons of Feeling. New York 1987.

Berleant, Arnold: Aesthetics Beyond the Arts. New and Recent Essays. New York 2016.

Cavell, Stanley: Themes out of School. Effects and Causes. Chicago 1988.

Cavell, Stanley: Conditions Handsome and Unhandsome. The Constitution of Emersonian Perfectionism. Chicago 1990.

Danto, Arthur C.: The Transfiguration of the Commonplace. A Philosophy of Art. Cambridge, Mass. 1981.

Danto, Arthur C.: After the End of Art. Contemporary Art and the Pale of History. Princeton 2014.

Dewey, John: Kunst als Erfahrung. Frankfurt a. M. 1980 (engl. 1934).

Emerson, Ralph Waldo: Art [1841]. In: Alfred R. Ferguson, Jean Ferguson Carr (Hg.): The Essays of Ralph Waldo Emerson. Cambridge, Mass. 1987.

Goodman, Nelson: Languages of Art. An Approach to a Theory of Symbols. 1968.

Goodman, Nelson: Ways of Worldmaking. Cambridge, Mass. 1978.

Goodman, Nelson: Of Mind and Other Matters. Cambridge, Mass. 1984.

James, William: The Principles of Psychology [1890]. Cambridge, Mass. 1983.

Margolis, Joseph: Culture and Cultural Entities. Toward a New Unity of Science. Dordrecht 1984. 
Margolis, Joseph: On Aesthetics. Boston 2008.

Margolis, Joseph: Selves and Other Texts. The Case for Cultural Realism. Pennsylvania 2010.

Margolis, Joseph: Toward a Metaphysics of Culture. New York 2016.

Pappas, Gregory: John Dewey's Ethics. Democracy as Experience. Bloomington 2008.

Peirce, Charles Sanders: The Essential Peirce. Selected Philosophical Writings 1893-1913. Bd. 2. Bloomington 1998.

Rorty, Richard: Contingency, Irony, and Solidarity. Cambridge, Mass. 1989.

Shusterman, Richard: Pragmatist Aesthetics. Living Beauty, Rethinking Art. Lanham 2002.
Shusterman, Richard: Aesthetics. In: John R. Shook, Joseph Margolis (Hg.): A Companion to Pragmatism. Malden 2006, 352-360.

Shusterman, Richard: Body Consciousness. A Philosophy Of Mindfulness And Somaesthetics. Cambridge 2008.

Shusterman, Richard: The Pragmatist Aesthetics of William James. In: British Journal of Aesthetics 51/4 (2011), 347361.

Stroud, Scott R.: John Dewey and the Question of Artful Criticism. In: Philosophy and Rhetoric 44/1 (2011), 27-51. 